\title{
Die Beziehungen zwischen E. F. v. Schlotheim und D. L. G. Karsten (nach den Briefen von Karsten an Schlotheim)
}

\author{
Günter Hoppe ${ }^{1}$
}

Mit 2 Abbildungen

\section{Zusammenfassung}

Ein anonymes Angebot zum Mineraltausch von 1790 und anschließende, bisher unbekannte Briefe leiteten die Beziehungen von D. L. G. Karsten zu E. F. v. Schlotheim ein. Der später berühmte Paläontologe Schlotheim zeigte sich noch ganz als mineralogischer Sammler. Nach dem Studium Schlotheims an der Bergakademie in Freiberg setzten sich die Kontakte fort. Sie lassen Karstens lebhaftes Interesse an Schlotheims beginnenden paläontologischen Arbeiten erkennen.

\begin{abstract}
So far unknown letters from D. L. G. Karsten to E. F. v. Schlotheim and an offer to change minerals from the year 1790 stated the connection between both men. The later famous paleontologist Schlotheim was a mineralogical collector at that time. After studying in Freiberg, Schlotheim developed paleontological interests. In this Karsten confirmed him.
\end{abstract}

\section{Einleitung}

Ernst Friedrich Freiherr von Schlotheim (1764-1832) war ein hoher Beamter in der Verwaltung des Herzogtums Sachsen-Gotha und zugleich ein bedeutender Paläontologe, der sich sowohl durch seine Werke als auch durch seine Sammlung berühmt gemacht hat. Da er sich neben den tierischen auch sehr früh der pflanzlichen Versteinerungen angenommen hat, gilt er als der Schöpfer der Phytopaläontologie. Für Berlin hat er die spezielle Bedeutung, dass seine paläontologische Sammlung nach seinem Tode durch Kauf an die Universität Berlin gelangte, wo sie einen höchst wertvollen Zuwachs zu den bereits vorhandenen Beständen des Mineralogischen Museums bildete. Zusammen mit Schlotheims Hauptwerk „Die Petrefaktenkunde auf ihrem jetzigen Standpunkt" (Schlotheim 1820) diente die Sammlung bald nach der Erwerbung dem damaligen "Gehilfen“ Friedrich Quenstedt als wesentlicher Leitfaden bei der Erarbeitung des Kataloges der Versteinerungssammlung dieses Museums. ${ }^{2}$ Noch heute sind die Originale zu den von Schlotheim beschriebenen Arten ein höchst wertvoller Bestand des jetzigen Paläonto- logischen Institutes im Museum für Naturkunde der Humboldt-Universität.

Die Entwicklung der geowissenschaftlichen Interessen Schlotheims begann, wie Oschmann (1964) und Martens (1982) darstellten, schon sehr früh. Schlotheims Vater war als herzoglich Sachsen-Gothaischer Regierungsrat seit 1776 Amtshauptmann in Gräfentonna. Von dort war es nicht weit bis zu den Travertingruben an der Fahner Höhe bei Burgtonna, wo Vater und Sohn Minerale und Konchylien sammelten. Am Gymnasium sammelte Schlotheim weiter und konnte dies auch von 1782 an neben dem Jurastudium an der Universität Göttingen, das er für seinen späteren Beruf betrieb, fortsetzen. Seine Neigungen zu den Geowissenschaften wurden durch die Vorlesungen des Göttinger Professors der Medizin und Naturkunde, Johann Friedrich Blumenbach, unterstützt. Nach diesem Studium kehrte er nach Gräfentonna zurück. Dort beschäftigte er sich mineralogisch-paläontologisch weiter und konnte schließlich aufgrund eigener Studien eine „Mineralogische Beschreibung der unteren Herrschaft Tonna“ (Schlotheim 1791) herausbringen. Schlotheim strebte die Tätigkeit in der Gothaischen Verwaltung auf berg- und hüttenkundli-

\footnotetext{
${ }^{1}$ Wilhelm-Wolff-Str. 65, D-13156 Berlin; - Museum für Naturkunde, Institut für Mineralogie, Invalidenstr. 43, D-10115 Berlin, Germany.

Erhalten Januar 2001, angenommen Mai 2001

2 Durch diese Arbeit gelang es F. Quenstedt, der bis dahin schon mineralogisch-kristallographische Erfolge erzielt hatte. sich paläontologisch in solchem Maße zu bilden, dass er anschließend den Ruf an die Universität Tübingen erhielt.
} 
chem Gebiet an. wofür er sich an der Bergakademie in Freiberg auszubilden gedachte. Ein Gesuch an den Gothaer Herzog um finanzielle Unterstützung für das recht teure Studium hatte Erfolg und er konnte sich vom 27.6.1791 an bis Februar 1792 in Freiberg dem Bergfach widmen. wo zur gleichen Zeit u. a. auch Alexander v. Humboldt unc Leopold v. Buch studierten (Wagenbreth 1967). Seine Lehrer waren dort. wie er später in einer Widmung ${ }^{3}$ angab. Oberberghauptmann F. W. H. v. Trebra. Berghauptmann J. F. W. T. v. Charpent er und Bergrat A. G. Wemer. Hier erreichte ihn roch 1791 die Nachricht. dass er als Kammerassessor in den Gothaischen Staatsdienst übernommen wurde. Von da an durchlief er cine erfolgreiche Karriere und stieg bis zum Präsidenten des Kamrierkollegiums. auch zum Oberhofmarschall und Oberaufseher des herzoglichen Museums auf. Daneben konnte er sich weiterhin erfolgreich wissenschaftlich betätigen. Sein Interesse verlagerti sich dabei immer stärker auf die Versteinerung $2 n$ und speziell auf die Spuren fossiler Pflanzen.

Nach diese." Charakterisierung von v. Schlotheim ist es noch notwendig. den fast gleichaltrigen Dietrich Ludwig Gustav Karsten (1768-1810) vorzustellen. Dieser gelangte schon mit 14 Jahren in eine gezielte geowissenschaftliche Ausbildung. da er. gefördert durch den verdienstvollen preußischen Bergbauminister F. A. Frhr. v. Heinitz (Heynitz), als preußischer Bergeleve auf dic Bergakademie Freiberg gesandt wurde. Er entwickelte sich dort zu einem überzeugten Schüer A. G. Werners und verteidigte dessen Lehre bereits frühzeitig vehement in Publikationen (Hoppe 1985). Vor seinem Abgang aus Freiberg m Jahre 1786 arbeitete er noch im Auftrage Werners an dem Katalog der bekannten Mineralsammlung des Freiberger Berghauptmanns Pabst von Ohain, den Werner erst 5 Jahre später herau!brachte. Karsten ging dann zum Studium an die Universität Halle. wo er im Jahre 1789 mit ein $2 r$ mineralogischen Arbeit promovierte und konnte noch im gleichen Jahr seine Fähigkeiten durch den Katalog der Sammlungen des verstorb nnen Leipziger Professors G. N. Leske nachweisen (Karsten 1789). Im gleichen Jahr berief ihn der Minister Heinitz nach Berlin als Bergassessor und beauftragte ihn mit der Lehre der $\mathrm{M}$ neralogie an der Berliner Bergakademie und $m t$ der Aufsicht über das Mincralienkabinett. Die; hatte sich notwendig gemacht. da der schwedische Mineraloge J. J. Ferber, den Heinitz als Nachfolger des Gründers der Bergakademie C. A. Gerhard eingesetzt hatte, durch schwere Erkrankung ausgefallen war. Als dieser 1790 starb, erhielt Karsten neben seinen sonstigen Dienstaufgaben die genannten Aufträge für dauernd. Karsten gab seine Mineralsammlung in das Königliche Mineralienkabinett und verzichtete auf privates Sammeln. Dieses freundschaftliche Verhalten brachte ihn in Verbindung mit A. v. Humboldt und L. v. Buch, die ihre Aufsammlungen auch dort niederlegten. Mit Karsten war die Lehre Werners an der Bergakademie Berlin eingezogen, die Karsten jedoch in chemischer und physikalischer Hinsicht weiterentwickelte. Vor allem machte er sich durch die Einführung der Kristallographie des Franzosen R. J. Haüy in Deutschland verdient, wofür er den Leipziger Physiker C. S. Weiss hinzuzog. Karsten verstarb sehr früh im Alter von 42 Jahren, kurz nach seiner Ernennung zum obersten Beamten des preußischen Berg- und Hüttenwesen. Sein Nachfolger an der im gleichen Jahr 1810 gegründeten Berliner Universität wurde C. S. Weiss (Hoppe 1999, 2000, 2001).

\section{Die ersten Kontakte Schlotheims zu Karsten}

Den Anlass für die Bekanntschaft von Karsten und Schlotheim gab ein anonymes Angebot zum Mineraltausch, das im Intelligenzblatt der Allgemeinen Literaturzeitung am 13.2.1790 erschienen ist (Anonym 1790). Es erregte das Interesse Karstens. dem daran lag, alle neuen Mineralfunde für das ihm vor kurzem anvertraute Mineralienkabinett zu beschaffen. Das Angebot lautete wie folgt:

\footnotetext{
..Auch in Thüringen hat man vor kurzen Gyps und Quarz in Verbindung angetroffen. und da er sich sehr von dem Spanischen zu Compostella sowohl. als auch von dem, den $\mathrm{Hr}$. Lasius neuerlich entdeckt, und Hr. Westrumb zerlegt hat, untorscheidet. so wird hoffentlich seine Bekanntmachung und nähere Beschreibung jedem Mineralogen angenehm seyn. Hier liegen kleine sehr reine Quarzkrystalle, welche gewöhnlich sechsseitig säulenförmig. selten doppelt pyramidalisch krystallisirt sind. allenthalben in der Gypsmasse zerstreut und werden fest von ihr umschlossen: daher scheinen sie mir die Aufmerksamkeit der Mineralogen besonders zu verdienen. Diuser fleischrothe faserige Gyps lag nicrenweise in grauen Letten. der in der dasigen Gegend fast ohne Ausnahme die übrigen Schichten des Gypsflözes bedeckt, und es war sonderbar. dass er sich nur aul eincm Fleck von wenig Quadratruthen fand. Ohngcachtet der mühsamsten Nachsuchungen war er nirgend in der umliegenden Gegend, wo das Gypstlötz allenthalben zum Vorschein kam. wieder auf-
} 
zufinden und das Ganze wurde daher bald erschöpft. Viele Stuffen sind gleichsam mit Quarzkrystallen gespickt, eben so reichhaltig auf dem Bruche, und die Krystalle stechen wegen ihrer vorzüglichen Rcinheit so schön gegen die Fleischröthe des Gypses ab, dass sie in jedem Cabinette eine Zierde ausmachen werden. Um $\mathrm{zu}$ verhüten, dass der Besitz dieses Gypses den Liebhabern nicht durch Mineralicnhändler erschwort werde, biete ich jedem Liebhaber den Tausch gegen andere Mineralien an. Es versteht sich, dass sie ausgesuchte Stücke postfrey überschicken, und sie womöglich der Bestellung gleich beyfügen. Unter der Adresse an Hrn. Sekretair Wefeld zu Gräfentonna uber Gotha bitte ich sie abgehen zu lassen. und ungesäumt werde ich dafür ausgesuchte Gypsstücke überschicken. Wahrscheinlich wird dieser Gyps glciches Schicksal mit mehrern Mineralien haben, die bald nach ihrer Entdeckung erschöpft wurden, und daher in der Folge gar nicht mehr zu bekommen seyn."

Dieses Tauschangebot beantwortete Karsten am 24. 4. 1790 an die angegebene (Deck-)Adresse und erklärte sein Interesse an dem Tausch, wofür er mit Genehmigung seines „Chefs, des Herrn Ministers v. Heinitz, Exzellenz", schlesische Chrysoprase und Opale anbieten könne. Weil es sich aber um eine königliche Sammlung handle, mit der der Tausch geschehen solle, müsse zuerst die Gegenseite das angebotene Material schicken, und zwar an die Adresse: ${ }^{4}$

„An ein Hohes Berg- und Hütten-Departement des Königl. Preuß3. Gcneral-Ober-Finanz-Kriegs-und Domainen-Directorii zu Berlin, abzugeben an den H. Asscssor Karsten in dem Hause des H. Staatsministers von Heinitz Exzellenz."

Hiernach entstand ein Briefwechsel und der Autor des anonymen Tauschangebots meldete sich. Es war E. F. v. Schlotheim aus Gräfentonna. Da nur die Briefe Karstens überliefert sind, erfährt man dies aus Karstens nächstem Brief vom 15. 5. 1790, wonach Schlotheim eine Sendung mit den genannten Quarzen in Gips sowie weiteren neuen Funden versprach. Schlotheim wünschte außer den von Karsten angebotenen Tauschmineralen noch andere, darunter C. A. Gerhards Olivenerz und „Xisirten"5 Speckstein von Jonsbach, die Karsten aber, wie er schreibt, nicht senden kann, da es diese Minerale dort gar nicht gibt, denn das Erste ist ein Schmelzprodukt und das Zweitc ist kein Speckstein, sondern eine Hornblendevarietät. Karsten fügt hinzu:

„Ich muss aber recht sehr bitten, diese Ansichten über das Olivenerz und den Schlesischen Xisirten Spekstein bloß für sich zu behalten, da ich nicht gern das gute Vernchmen stören möchte, welches zwischen $\mathrm{H}$. Gerhard und mir jczt herrscht. In öffentlichen Schriften sage ich meine Meinung durchaus frei heraus, sic mag auch gegen jemand gerichtet sein, wer es wolle; aber wenn dergl[eichen] Widerlegungen aus Briefen bekannt gemacht werden, so kann es leicht das Ansehn gewinnen, als wollte man heimlich jemandes wahre oder vermeintliche Verdienste zu schmälern suchen. und das ist nicht meine Sache." ${ }^{* 6}$

Nachdem dann eine Mineralsendung und ein Brief von Schlotheim eingetroffen waren, meldet sich Karsten am 14.6.1790 und teilte mit, dass er nun die versprochenen Chrysoprase von Kosemitz in Schlesien sendet. Er betonte dabei, es würde sich um ,vorzüglich instruktive Stücke“ handeln, die besonders ausgewählt seien. da er es „mit einem Kenner und nicht mit einem bloBen Liebhaber zu tun habe", auch wären die Stücke an Ort und Stelle sehr teuer, da Kosemitz der einzige Ort auf der Welt sei, wo Chrysopras vorkommt. Man erfährt auch, dass er an der Sendung Schlotheims manches bemängelte, die Stücke hätten teilweise mehr frischen Bruch und größere Kristalle haben können, auch war nicht alles Versprochene dabei, aber er würde sich damit begnügen, da Schlotheim für die Zukunft weitere Sendungen versprochen hätte. Was cr jedoch ernsthaft rügte, war das Fehlen der ..genauen Bestimmung des Geburtsortes", die bloße Angabe der Provinz Thüringen sei viel zu allgemein. ${ }^{7}$ Weiterhin tauschte Karsten in dem Brief noch verschiedene Mitteilungen über einzelne Minerale aus.

Nach Rückkehr von einer mehrmonatigen Inspektionsreise fand Karsten dann Briefe Schlotheims vom Juli und September und cine Sendung vor und antwortete wegen Arbeitsüberlastung erst am 15.1.1791. Er wusste nicht recht, was er schicken kann und hoffte. dass der bevorstehende Kauf der über 12000 Stück umfassenden großen Mineralsammlung seines verstorbenen Vorgängers J. J. Ferber Dubletten liefern wird, die er zum Tausch anbieten kann. Weiter heißt es:

„Doch ist dies unter Jahr und Tag deswegen nicht möglich. weil es uns noch an Platz für dies beträchtliche Kabinet fehlt. davon selbst einige 20 Kisten noch nicht ausgepakt sind. Es

\footnotetext{
${ }^{4}$ Die wiedergegebenen Auszüge und Zitate stammen aus fünf Briefen Karstens an Schlotheim aus den Jahren 1790 und 1791, die sich im Handschriftenbestand des Institutes für Mineralogie der Humboldt-Universität Berlin befinden (MfN: c). Herrn Dr. Gert Wappler wird für die Vermittlung der Briefe gedankt. - In allen Zitaten aus Bricfen Karstens wird dessen Orthographie beibehalten. Unterstreichungen stammen von Karsten.

$\hat{\mathrm{X}}=$ Abkürzung für Kristall.

${ }^{6}$ Karstens Verhältnjs zu Gerhard war gespannt, da Letzterer bis vor vier Jahren noch an der Bergakademie gelehrt hatte (und scitdem als höchster Beamter unter dem Minister in der Berg- und Hüttenverwaltung weiter tätig war), und Karsten teilweise merklich andere wissenschaftliche Ansichten vertrat.

${ }^{7}$ Diese Unsitte war (und ist auch noch heute) bei Mineralsammlern weit verbreitet. Sie sollte andere Sammler durch Geheimhaltung an der Ausbeutung des Fundortes hindern.
} 
wird daher diesen Winter bloß verschlossen in einen Saal gestellt, und kann ni ht eher ausgepakt oder geordnet werden. bis mir nicht ein hinlänglich großer Raum dazu angewiesen ist. Ich sehe dabei freilich einer erstaunlichen Arbeit entgegen."

Der Ton des Briefwechsels hat sich inzwischen verändert, offensichtlich ist nun gegenseitiges Verständnis und Vertrauen cingetreten. Es folgen noch Mitteilungen verschiedener Neuigkeiten auf dem Gebiet de:s Mineralsammelns (offensichtlich auch in den Briefen Schlotheims). Karsten teilte sogar mit, dass er an den Experimenten des Berliner Chemike:s M. H. Klaproth zu dem damals aktuellen chemischen Problem der Reduzierung der sog. einfachen Erden mitarbeitet.

Den letzten Brief dieser Serie schrieb Karsten am 26.3.1791 und antwortete zunächst auf eine für ihn überraschende Mitteilung Schlotheims:

..Ihr lezter angen hmer Brief vom 12. Febr. bestätigt es mir aufs Neue Ihren yroßen. sehr liebenswürdigen Eifer für das mineralogische Studium. und ich freue mich über Nichts mehr, als dass Sir nach Freiberg gehen. und selbst aus der Quelle schöpfen wollen. Diesen Vorsaz hat Ihnen ein guter Genius eingegebe . Versäumen Sie ja nicht ihn auszuführen (Abb. 1).

Selbst aus Inter zsse für unser hiesiges jezt sehr großes $\mathrm{Mi}$ neralienkabinet wünsche ich es: denn ob wir zwar prächtige große Sachen aus Sachsen in Ueberfluss haben. so fehlt es uns doch noch zum Theil an den gemeinsten Dingen. und Sie glauben nicht. wie schwer es fält, die H[erren] Sachsen dazu zu bringen. dass Sie einem deren schikken. Sobald Sie in Freiberg sein werden. will ich Ihnen ganz frei die Dinge benennen. welche ich noch zu haben wünsche."

Karsten begrüßte also die Absicht Schlotheims, an der Bergakademie Freiberg zu studieren, und ergriff in seinem Bestreben, das Berliner Mineralienkabinett zu vervollständigen, sofort die sich dadurch möglicherweise bietende Gelegenheit für künftige Mineralbeschaffungen. ${ }^{8}$ Einen direkten Einfluss Karstens auf den Entschluss Schlotheims hat es nach den vorliegenden Briefen übrigens nicht gegeben. Im Brief vom 26. 3. 1791 folgt weiter:

..Aber ehe Sic nach dorthin gehen, wünsche ich wohl aus Ihrer Gegend Folgendes für die hiesige Akademische Sammlung zu erhalten: 1. Honigstein von Artern, den Sie aus meinem Mus[eum] Leskean[um] [Karsten 1789| hinlänglich kennen werden. 2. Gipserde oder so genanntes Himmelsmehl. 3. Mergelerde oder so genanntes Aschengebirge. Ich kann nicht glauben. dass es Ihnen schwer werden sollte, diese Sachen zu bekommen."

Es folgen dann noch Bemerkungen zu neuen Mineralfunden und -erwerbungen ${ }^{9}$ und Karsten schließt in der Hoffnung, noch einen Brief von Schlotheim vor seiner Abreise nach Freiberg zu erhalten. ${ }^{10}$

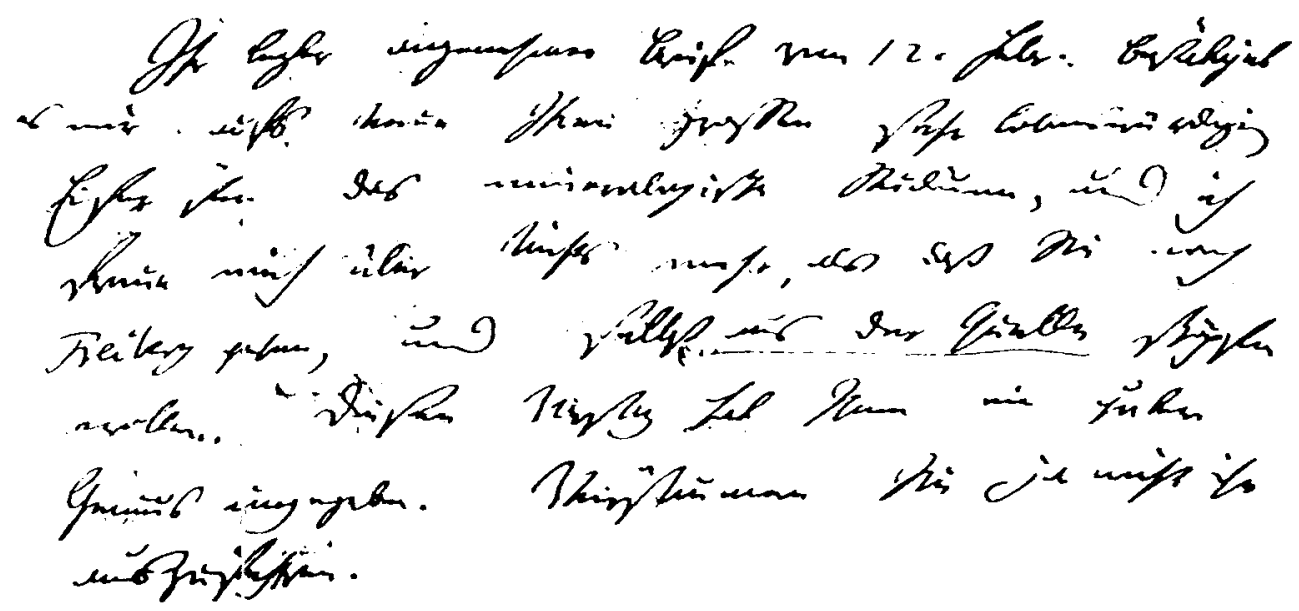

Abb. 1. Ausschnit aus dem Brief D. L. G. Karstens an E. F. v. Schlotheim vom 26. 3. 1791.

Fig. 1. Excerpt from a letter of D. L. G. Karsten to E. F. 、. Schlotheim from 26. 3. 1791.

\footnotetext{
Karsten machte etliche Jahre später noch einmal einen Versuch dieser Art, als ein preußischer Bergeleve, C. F. v. Klass. zum weiteren Studium nach Freiberg gesandt wurde. Diesem trug Karsten auch auf. sächsische Minerale zu beschaffen. Klass machte dies Müh: und beklagte die Schwierigkeiten. Was Karsten in einem Brief an ihn vom 2.2. 1808 wie folgt beantwortete: Allerdings ist es schwierig. sonst hätten wir ja die Exemplare längst. Aber eben deswegen habe ich Ihnen den Auftrag gegeben, weil Sie an fer Quelle und unterrichtet sind. Prachtstükke erhält man überall für Geld. instructive Kennzeichenstükke nur durch beharrliches Suchen und Bemühen. Deshalb liegt mir so viel daran."

" Darunter bet ndet sich die folgende ungewöhnliche Erwerbung:..Auch habe ich für unser Kabinet vor Kurzem eine sehr wichtige Acquisit on durch ein großes Stük ächten Buttermilcherzes vom Harz gemacht, welches daselbst im Jahre 1618 gebrochen. und jezt nur dem Nahmen nach. noch dort rorkommt: es kostete indessen 25 rthl [Reichsthaler]." - Karsten brachte für ein solches h storisches Sammlungsstück trotz des außerordentlich hohen Betrages Verständnis auf. Dieses Stück blieb erhalten und kon te einer modernen Untersuchung unterzogen werden. wodurch es als feinstkörniges Gemenge von Chlorargyrit (Silberchlorif) und Tonmineralen identifziert wurde (Hoppe \& Damaschun 1986).

16) Wie spätere Briefe zeigen. hat sich Schlotheim in Freiberg intensiv mit der Lehre Werners vertraut gemacht und das Erkennungssyster 1 Werners für Minerale getreu nach dem Schematismus seines Lebrers angewandt (Schlotheim 1810e).
} 


\section{Die späteren Briefe Karstens an Schlotheim}

Die weiteren überlieferten Briefe Karstens an Schlotheim setzen erst im Jahre 1802 ein. Es sind vier Briefe, die bis zum Jahre 1810, dem Todesjahr Karstens, reichen. ${ }^{11}$ Inzwischen war Schlotheim in Freiberg gewesen, auch eine erste paläobotanische Publikation von ihm war erschienen (Schlotheim 1801). In Berlin war für das Königliche Mineralienkabinett im Jahre 1801 eine sehr verbesserte Unterbringung im Neubau für die Münze geschaffen worden, sodass es den Charakter eines Museums erhalten hatte, und Karsten war 1797 zum Oberbergrat im Bergund Hütten-Departement aufgestiegen (Hoppe 1999).

Der erste Brief, den Karsten am 2.2.1802 geschrieben hat, besitzt einen stärker wissenschaftlichen Charakter als die früheren Briefe, indem u. a. über eine Elemententdeckung und über chemische Feldspatanalysen mit eingehender Diskussion der konkreten Ergebnisse gesprochen wird. Aber auch in ihm sind Neuigkeiten des Mineralsammelns und -handels enthalten. Im Übrigen lässt der Inhalt erkennen, dass der Kontakt zwischen Karsten und Schlotheim keineswegs abgerissen war. Es müssen weitere Briefe existiert haben und es ist auch zu wechselseitigen persönlichen Kontakten in Gotha und in Berlin gekommen. Der Besuch Schlotheims im Königlichen Mineralienkabinett in Berlin, bei dem Schlotheim Palmenstücke und Abdrücke gesehen hat (Schlotheim 1804: 27), dürfte aber bereits vor 1801 stattgefunden haben, da sich dort in dem 1801 begonnenen Besucherbuch keine Eintragung Schlotheims befindet.

Den zweiten Brief sandte Karsten am 3.12. 1803. Er wird hier vollständig wiedergegeben:

„Unlängst erfahre ich dass der Fasrige Schwarz-Eisenstein zu Brotterode bei Schmalkalden brechen soll. Sie wohnen diesem Orte fast 40 Meilen näher als ich, und haben dort ohne Zweifel einige Bekanntschaft. Dürfte ich Sic bitten, gegen dankbare Erstattung jeder Auslage, ein deutliches Stük oder mehrere davon zu verschaffen und mit dor Post, aber recht wohl verpakt herzuschikken? - Es ist mir äußerst unange- nehm dass unsere K[önigliche] SammI[ung] noch gar nichts davon besitzt, besonders meiner Vorlesungen wegen.

Meine Sehnsucht nach Ihren treffl[ichen] Ausarbeitungen. in Betreff der Farnkraut p. Abdrükke, ist noch immer unbefriedigt. Darf ich nicht hoffen, dass die Ostermesse etwas davon mitbringen wird?

Wo lebt H[err] v. Hoff?? Vor fast 2 Jahren schikte ich ihm einen Aufsatz fürs Magazin. der damahls manchen Freunden der Krystallogr[aphie] vielleicht willkommen gewesen. jetzt aber nicht mehr à l'ordre du jour sein würde. ${ }^{12}$ Könnte ich ihn gelegentlich zurück erhalten? Aber weit wichtiger ist mir die Frage, wie es Ihnen u. Ihrem Freunde geht? Ob Sie das nützliche Journal nicht fortsetzen werden? Ob ich noch auf ihr Wohlwollen, und zuweilen auf Ihre lehrreichen Briefe rechnen kann? Hierüber geben Sie mir wenigstens cine gütige Auskunft, und halten Sie sich versichert von der Hochachtung und den freundschaftlichsten Gesinnungen Ihres gehorsamen Dieners Karsten."

Im Gegensatz zu diesem Brief mit seinen Wünschen und Fragen hat der Dritte vom 16.4.1804 wieder mehr einen Dialogcharakter. Karsten erwähnt, dass er eine kleine Mitteilung Schlotheims über Edelsteine in der Berliner Gesellschaft naturforschender Freunde vorgetragen hat, und geht auf die Bedingungen für die Aufnahme von Mitgliedern in diese Gesellschaft ein. da er gern Schlotheim dazu verhelfen will. ${ }^{13} \mathrm{Er}$ nimmt auch an Schlotheims Spezialität, der Beschäftigung mit Pflanzenabdrücken, lebhaften Anteil und freut sich über Folgendes:

„Im Messkatalog [der Leipziger Buchmessel finde ich Gottlob ihr Werk von den Abdrükken ${ }^{14}$ angekündiget. Da Sie noch immer mehrere erhalten, so haben wir wohl von Zeit zu Zeit Nachträge zu hoffen? Gerne will auch ich Ihnen Bciträge dazu liefern. Besitzen Sie z. B. schon die sonderbaren geschlängelten Pflanzen Abdrükke in dem Kupferschiefer von Rothenburg?*

Aus den Akten der Gesellschaft naturforschender Freunde zu Berlin ist zu erfahren, dass Karsten am 13.1.1807 den Vorschlag gemacht hat, Schlotheim als auswärtiges Mitglied zu wählen, was 2 Wochen später einstimmig erfolgt ist, übrigens gleichzeitig mit der Wahl des Gothaischen Legationsrates K. E. A. v. Hoff (MfN a, VI, S. 396, 398). Die im Zusammenhang hiermit gewechselten Briefe sind nicht überliefert.

Später hat Karsten noch mindestens weitere zwei Briefe erhalten, aus denen er in der Ge-

\footnotetext{
11 Diese Briefe befinden sich in der Forschungsbibliothek Gotha (FbG). Für die freundliche Gestattung ihrer Verwendung wird auch an diescr Stelle gedankt.

12 Karsten hält das Manuskript inzwischen nicht mehr für aktuell. Es war das Manuskript eines Vortrages von Karsten über Haüys Methode der Kristallographie, das in dem von v. Hoff herausgegebenen Magazin für die gesamte Mineralogie, Gcognosie und mineralogische Erdbeschreibung erscheinen sollte. Die Zeitschrift stellte aber schon nach den vier Heften ihres ersten Jahrgangs 1801 das Erscheinen cin, sodass das Manuskript ungedruckt blieb. - Das Manuskript gelangte später nach Bonn und ist dort in der Handschriftensammlung der Universitätsbibliothek Bonn vorhanden. Im Jahre 2000 wurde es auszugsweise in einem Artikel zur Geschichte der Geowissenschaften im Berliner Museums für Naturkunde veröffentlicht (Hoppe 2000)

${ }_{13}$ Karsten gehörte in dieser Gesellschaft dem engeren Krcis der ordentlichen Mitglieder an, die das Direktorium der Gesellschaft bildeten. Er vermittelte die Annahme dieses und eines weiteren Beitrages Schlotheims für die Zeitschrift der Gesellschaft (Schlotheim 1807a, b). Von beiden sind die Manuskripte erhalten geblieben (MfN b, Blatt 3-5).

${ }^{14}$ Schlotheim 1804
} 


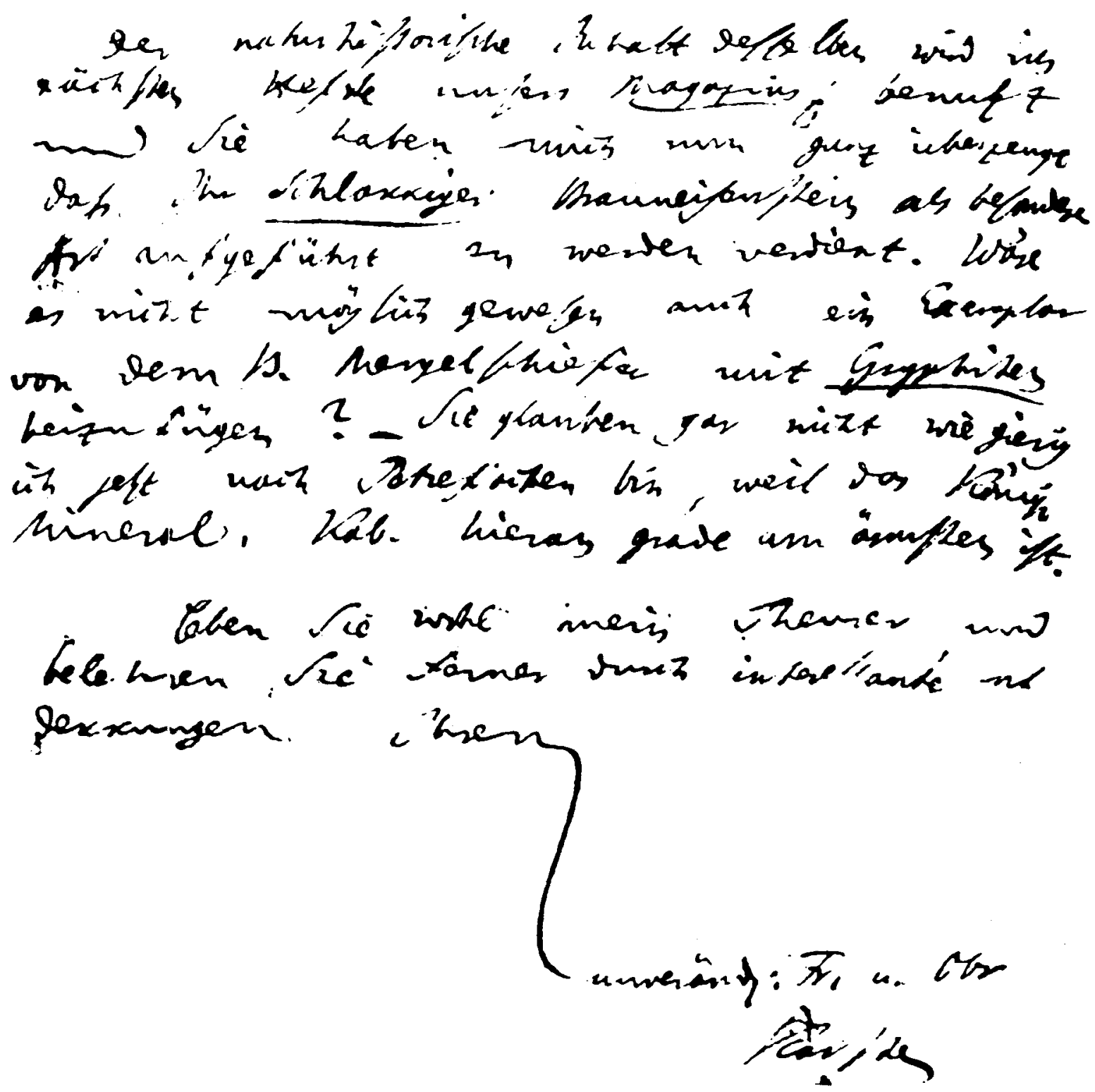

Abb. 2. Ausschni t aus dem Brief D. L. G. Karstens an E. F. v. Schlotheim vom 10.2. 1810.

Fig. 2. Excerpt from a letter of D. L. G. Karsten to E. F. v. Schlotheim from 10. 2. 1810.

sellschaft vor.as und zum Druck gab (MfN c. Blatt 6-8, Schlotheim $1810 \mathrm{a}-\mathrm{c})$. Dies bestätigte Karsten in einem Brief vom 10.2.1810, in dem es unter ande: em heißt:

..Der naturhistotische Inhalt desselben wird im nächsten Hefte unsers Ma \azins benutzt. [...] Wäre es nicht möglich gewesen auch eir Exemplar von dem B[ituminösen] Mergelschicfer [Kupfers shiefer] mit Gryphiten [Muscheln] beizufügen? - Sie glauben gar nicht. wie gierig ich jetzt nach Petrefacten bin. weil das Königl. Mineral[ien] Kab[inett] hieran grade am ärmsteı! ist.

Leben Sie wohl mein Theurer und belehren Sie ferner durch interessant : Entdekkungen." (Abb. 2)
Das ist der Schluss der Korrespondenz, da Karsten. der in den letzten Jahren mehrmals schwer erkrankt war, am 20.5.1810 starb. Damit wurde zwar der Kontakt Schlotheims mit dem Königlichen Mineralienkabinett in Berlin, das im gleichen Jahr als Mineralogisches Museum der neu gegründeten Berliner Universität eingegliedert wurde, nicht völlig beendet, auch der Kontakt zu der Gesellschaft naturforschender Freunde nicht. Aber die Beziehungen Schlotheims zu dem Nachfolger Karstens an der Universität, dem

15 A. v. Humboldts und L. v. Buchs Fürsorge für die Berliner Sammlung knüpft an das Vertrauensverhältnis an, das zwischen ihnen und Karsten bestanden hatte. Karstens selbstlose Führung der ihm anvertrauten offentlichen Sammlung hatte sie von Beginn ihrer geowissenschaftlichen Betätigung an bewogen. keine privaten Sammlungen anzulegen, sondern ibr Sammelgut in die von Karsten geführten Sammlung 7u geben (mit der Ausnahme der nach Paris und Madrid gegebenen Teile der amerikanischen Sammlung Humboldts), ohne dafür eine Bezahlung zu fordern. 
Kristallographen C. S. Weiss, entwickelten sich nicht so fruchtbar, wie sie zwischen ihm und Karsten bestanden hatten.

Es waren die beiden alten Freiberger Studienkollegen Schlotheims, A. v. Humboldt und L. von Buch, denen es nach dem Tode Schlotheims gelang, das preußische Kultusministerium dafür zu gewinnen, die bedeutende Petrefakten-Sammlung Schlotheims für die Universität Berlin anzukaufen (Schuster 1922). So fand schließlich Karstens Bestreben, das königliche Mineralienkabinett, das inzwischen als Mineralogisches Museum der Universität angehörte, durch Versteinerungen $\mathrm{zu}$ vervollständigen, in großem Maßstab seine Erfüllung. ${ }^{15}$ (s. S. 34)

\section{Archivalien}

MfN: Museum für Naturkunde der Humboldt-Universität zu Berlin. Historische Bild- und Schriftgutsammlungen.

a: Bestand: GnF, Tagebücher der Gesellschaft naturforschender Freunde zu Berlin.

b: Bestand: GnF, Briefe von E. F. v. Schlotheim, Signatur: S. Schlotheim.

c: Bestand: Mineralogisches Institut, Briefe von D. L. G. Karsten an E. F. v. Schlotheim.

FbG: Forschungsbibliothek Gotha, Schloss Friedenstein. 4 Briefe von D. L. G. Karsten an E. F. v. Schlotheim aus den Jahren $1802,1803,1804$ und 1810, Signatur: Chart. B 1935 (3), Nr. 28-31.

\section{Literatur}

Anonym [Schlotheim, E. F. v.]. 1790. [Mittcilung über Quarz in Gipsgestein mit Tauschangebot]. - Intelligenzblatt der Allgemeinen Literatur-Zeitung, Nr. 22, 13. 2. 1790.

Hoppe, G. 1985. Dietrich Ludwig Gustav Karsten (17681810). Mineraloge und Bergbcamter in Preußen. - In: Prescher, H. (Hrsg.): Leben und Wirken deutscher Geologen im 18. und 19. Jahrhundert: 71-92. Deutscher Verlag für Grundstoffindustrie, Leipzig.

- 1999. Zur Geschichte der Geowissenschaften im Museum für Naturkunde zu Berlin. Teil 2. Von der Gründung der Bergakademic zur Gründung der Universität 1770-1810.

- Mitteilungen aus dem Museum für Naturkunde der Humboldt-Universität in Bcrlin, Geowissenschaftliche Reihe 2: 3-24.

- 2000. Zur Geschichte der Geowissenschaften im Museum für Naturkunde zu Berlin. Teil 3. Von A. G. Werner und R. J. Haüy zu C. S. Weiss - Der Weg von C. S. Weiss zum Direktor des Mineralogischen Museums der Berliner Universität. - Mitteilungen aus dem Museum für Naturkunde der Humboldt-Universität in Berlin. Geowissenschaftliche Reihe 3: 3-25.

- 2001. Zur Geschichte der Geowissenschaften im Museum lür Naturkunde zu Berlin. Teil 4: Das Mineralogische Museum der Universität Bcrlin unter Christian Samuel Weiss von 1810 bis 1856 . - Mitteilungen aus dem Museum für Naturkunde der Humboldt-Universität in Berlin, Geowissenschaftliche Reihe 4: 3-27.
Hoppe, G. \& Damaschun, F. 1986. Das historische Buttermilcherz von Adreasberg/Harz (BRD), ein Gemenge von Chlorargyrit und Schichtsilikaten. - Chemie der Erde 45: $147-158$.

Karsten, D. L. G. 1789. Museum Leskeanum. Vol. 2. Regnum minerale. [Nebentitel:] Des Herrn Nathanacl Gottfried Leske hinterlassenes Mineralienkabinct systematisch geordnet und beschrieben. 54 und 578 pp.. J. G. Müller, Leipzig.

Martens, Th. 1982. Ernst Friedrich von Schlotheim (1764-1832) - ein bedeutender Paläontologe der Goethezeit. $-($ Gothaer Museumsheft $=)$ Abhandlungen und Berichte des Museums der Natur Gotha 11: 5-22.

Oschmann, M. 1964. E. F. von Schlotheim. - Bergakademic 16: $444-448$.

Schlotheim. E. F. v. 1791. Mineralogische Beschreibung der unteren Herrschaft Tonna. - Mineralogische und bergmännische Abhandlungen (Hrsg. J. C. W. Voigt) 3: $182-200$.

- 1801. Abhandlung über die Kräuter-Abdrücke in Schieferthon und Sandstein der Steinkohlen-Formationen. - Magazin für die gesammte Mineralogie, Geognosic und mineralogische Erdbeschreibung (Hrsg. K. E. A. v. Hoff) 1: $76-95$.

- 1804. Beschreibung merkwürdiger Kräuter-Abdrücke und Pflanzen-Versteinerungen. Ein Beitrag zur Flora der Vorwelt. 1. Abtheilung. $68 \mathrm{~S}$, 14 Tafcln. Becker. Gotha. (Nachdruck: Gesellschaft für Geologische Wissenschaften der DDR, Berlin 1981).

- 1807a. Beobachtungen an einigen Ostindischen Edelsteinen [bricfliche Mitteilung von v. Schlottheim an Karsten. darin u. a. über Sapparit]. - Magazin der Gesellschaft naturforschender Freunde zu Berlin 1: 302-305.

- 1807b. Auszug aus einem Briefe des Herrn Vice-Präsidenten von Schlottheim an den Geh. Rath Karsten vom 28. 8. 1807 [betr. u. a. Bolus und Bergseife bei Waltershausen]. - Magazin für die neuesten Entdeckungen in der gesammten Naturkunde der Gesellschaft naturforschender Freunde zu Berlin 1: 305-6.

- 1810a, b, c. Auszüge aus Briefen des Herm Präsidenten von Schlotheim an den geheimen Rath Karsten. - Magazin für die neuesten Entdeckungen in der gesammten Naturkunde der Gesellschaft naturforschender Freunde zu Berlin 4: 74-78. - a) Erster Brief, Gotha 2. 3. 1809 [ohne Titel, betr. u. a. Muschelversteinerungen im Bituminösen Muschelschiefer]: 74-75. - b) Zweiter Brief. 16. 1. 1810 [ohne Titel, betr. Nachrichten uber eigene und fremde Verstcincrungsfunde und -erwerbungen]: 75-77. - c) Dritter Brief: Über eine neue Art von Brauneisenstein: Schlackiger Braun-Eisenstein [vom Sperrweger Hauptgang des Grubengebäudes vom Wolfsstieg bei Friedrichroda]: 77-78.

- 1820. Die Petrefaktenkunde auf ihrem jetzigen Standpunkte durch die Beschreibung seiner Sammlung versteinerter und fossiler Überreste des Thier- und Pflanzenreichs der Vorwelt erläutert. LXII, 437 pp.. Becker, Gotha.

Schuster, J. 1922. Aus unveröffentlichten Dokumenten zur Geschichte der Mineralogie, Geologie und Paläontologic. Historische Miniaturen. III. Ernst Friedrich von Schlotheim und der Übergang seiner Petrefaktensammlung an den preußischen Staat. In Degering. H., Christ, K. \& Schuster, J.: Aus der Handschriftenabteilung der Preukischen Staatsbibliothek. Breslauer, Berlin: 103-109.

Wagenbreth, O. 1967. Werner-Schüler als Geologen und Bergleute und ihre Bedeutung für die Gcologie und den Bergbau des 19. Jahrhunderts. - Freiberger Forschungshefte C 223: 163-178. 